\title{
Ground states of pseudo-relativistic boson stars under the critical stellar mass
}

\author{
Yujin Guo ${ }^{\text {a }}$, Xiaoyu Zeng ${ }^{\mathrm{b}}$ \\ ${ }^{a}$ Wuhan Institute of Physics and Mathematics, Chinese Academy of Sciences, Wuhan 430071, PR China \\ ${ }^{\mathrm{b}}$ Department of Mathematics, Wuhan University of Technology, Wuhan 430070, PR China \\ Received 3 June 2015; received in revised form 5 March 2017; accepted 7 April 2017 \\ Available online 19 April 2017
}

\begin{abstract}
We consider ground states of pseudo-relativistic boson stars with a self-interacting potential $K(x)$ in $\mathbb{R}^{3}$, which can be described by minimizers of the pseudo-relativistic Hartree energy functional. Under some assumptions on $K(x)$, minimizers exist if the stellar mass $N$ satisfies $0<N<N^{*}$, and there is no minimizer if $N>N^{*}$, where $N^{*}$ is called the critical stellar mass. In contrast to the case of the Coulomb-type potential where $K(x) \equiv 1$, we prove that the existence of minimizers may occur at $N=N^{*}$, depending on the local profile of $K(x)$ near the origin. When there is no minimizer at $N=N^{*}$, we also present a detailed analysis of the behavior of minimizers as $N$ approaches $N^{*}$ from below, for which the stellar mass concentrates at a unique point.

(c) 2017 L'Association Publications de l'Institut Henri Poincaré. Published by Elsevier B.V. All rights reserved.
\end{abstract}

Keywords: Ground states; Boson stars; Mass concentration; Critical mass

\section{Introduction}

We study ground states of pseudo-relativistic boson stars in the mean field limit, which can be described (cf. [5,9, 20]) by minimizers of the following variational problem

$$
e(N):=\inf \left\{\mathcal{E}(u): u \in H^{\frac{1}{2}}\left(\mathbb{R}^{3}\right) \text { and } \int_{\mathbb{R}^{3}}|u(x)|^{2} d x=N\right\}
$$

under the stellar mass $N>0$ of boson stars, where the pseudo-relativistic Hartree energy functional $\mathcal{E}(u)$ is defined by

$$
\mathcal{E}(u):=\int_{\mathbb{R}^{3}} \bar{u}\left(\sqrt{-\Delta+m^{2}}-m\right) u d x-\frac{1}{2} \int_{\mathbb{R}^{3}}\left(\frac{K(x)}{|x|} *|u|^{2}\right)|u|^{2} d x .
$$

\footnotetext{
E-mail addresses: yjguo@wipm.ac.cn (Y. Guo), xyzeng@whut.edu.cn (X. Zeng).
} 
Here the operator $\sqrt{-\Delta+m^{2}}$ is defined via its symbol $\sqrt{k^{2}+m^{2}}$ in Fourier space, which describes the kinetic and rest energy of a relativistic particle with rest mass $m>0$, and the symbol $*$ stands for the convolution on $\mathbb{R}^{3}$. Moreover, we always assume that the self-interacting potential $K(x)$ of boson stars satisfies $0<K(x) \leq K(0)=1$ in $\mathbb{R}^{3}$. From physical point of view, there are two typical types of such potentials: one is the Coulomb-type potential $K(x) \equiv 1$, and the other is the Yukawa-type potential $K(x) \equiv e^{-\mu|x|}$ with $\mu>0$. Our investigation of $e(N)$ is motivated by our recent series of works $[4,12-15]$, where we studied the minimization problem of the Laplacian type arising from attractive Bose-Einstein condensates (BEC). However, the analysis of $e(N)$ is more complicated in a substantial way, due to the nonlocal nature of the pseudo-differential operator $\sqrt{-\Delta+m^{2}}$, and the convolution-type nonlinearity as well.

In the recent works $[8-10,16,17,20]$ and references therein, the authors discussed $e(N)$ for the case of the Coulombtype potential, i.e., $K(x) \equiv 1$, where the existence, nonexistence, dynamics and some other analytic properties of minimizers for $e(N)$ were obtained. The existing results of analyzing $e(N)$ show that the problem (1.1) is related well to the following Gagliardo-Nirenberg type inequality

$$
\int_{\mathbb{R}^{3}}\left(\frac{1}{|x|} *|u|^{2}\right)|u|^{2} d x \leq \frac{2}{\|Q\|_{2}^{2}}\left\|(-\Delta)^{1 / 4} u\right\|_{2}^{2}\|u\|_{2}^{2}, \quad u \in H^{\frac{1}{2}}\left(\mathbb{R}^{3}\right),
$$

where $Q(x)=Q(|x|)>0$ is a ground state, up to translations and suitable rescaling (cf. [2,6,9]), of the fractional equation

$$
\sqrt{-\Delta} u+u-\left(\frac{1}{|x|} *|u|^{2}\right) u=0 \text { in } \mathbb{R}^{3}, \text { where } u \in H^{\frac{1}{2}}\left(\mathbb{R}^{3}\right) .
$$

Similar to that of $[3,6,7,11]$, here we define

Definition 1.1. If $Q(x)=Q(|x|) \in H^{\frac{1}{2}}\left(\mathbb{R}^{3}\right)$ is a positive solution of (1.4), and $Q(x)$ optimizes (1.3), we then say that $Q(x)$ is a ground state of (1.4).

Note from [1,6,9] that (1.4) admits ground states, and all ground states of (1.4) must be radially symmetric and nonincreasing. Therefore, one can define the nonempty set $\mathcal{G}$ by

$$
\mathcal{G}=\{Q(x)=Q(|x|)>0: Q(x) \text { is a ground state of }(1.4)\} .
$$

It thus follows from (1.3) and (1.4) that

$$
\|Q\|_{2}^{2}=\left\|(-\Delta)^{1 / 4} Q\right\|_{2}^{2}=\frac{1}{2} \int_{\mathbb{R}^{3}}\left(\frac{1}{|x|} * Q^{2}\right) Q^{2} d x \text { for all } Q \in \mathcal{G} .
$$

Moreover, one can derive from [6, Lemma 2.2] that there exists $C>0$ such that for all $Q(x) \in \mathcal{G}$,

$$
|Q(x)| \leq C(1+|x|)^{-4} \text { in } \mathbb{R}^{3},
$$

and

$$
\left(|x|^{-1} *|Q|^{2}\right)(x) \leq C(1+|x|)^{-1} \text { in } \mathbb{R}^{3} .
$$

Making full use of the above results, inspired by $[9,12,20]$ we first derive the following existence of a critical stellar mass $N^{*}$.

Theorem 1.1. Let $Q$ be a ground state of (1.4), and suppose that $m>0$ and

$$
K(x) \in C\left(\mathbb{R}^{3}\right) \text { satisfies } 0<K(x) \leq K(0)=1 \text { in } \mathbb{R}^{3} .
$$

Then,

(a). If $0<N<N^{*}:=\|Q\|_{2}^{2}$, and assume that

$$
\text { either } m>0 \text { is large sufficiently or } K(x) \geq O\left(|x|^{-\alpha}\right) \text { as }|x| \rightarrow \infty \text { with } 0<\alpha<1 \text {, }
$$

then there exists at least one minimizer for (1.1).

(b). If $N>N^{*}$, there is no minimizer for (1.1). 
Theorem 1.1 is extended essentially from those obtained in $[9,20]$ for the special case where $K(x) \equiv 1$. The assumption (1.10) is to ensure the estimate $e(N)<0$ (see Lemma 2.1), which seems essential to the existence of minimizers. Actually, if $K(x)$ decays fast enough as $|x| \rightarrow \infty$, for example $K(x)=e^{-\mu|x|}$ with $\mu>0$, we may have $e(N)=0$ at least for both small $N>0$ and $m>0$, in which case minimizers may not exist. ${ }^{1}$ In view of Theorem 1.1, we are next concerned with the existence of minimizers at the critical stellar mass $N=N^{*}\left(=\|Q\|_{2}^{2}\right)$, in which case we can prove the following results provided that $K(x)$ satisfies (1.11) below.

Theorem 1.2. Let $m>0$ and suppose $K(x)$ satisfies the assumptions (1.9) and (1.10). If there exists a constant $p>0$ such that

$$
K(x)=1-O\left(|x|^{p}\right) \geq 0 \text { as }|x| \rightarrow 0,
$$

then we have

(a). If $1<p<\infty$, there is no minimizer for (1.1) at $N=N^{*}$.

(b). If $0<p<1$, then there exists at least one minimizer for (1.1) at $N=N^{*}$.

(c). If $p=1$ and $\liminf _{|x| \rightarrow 0} \frac{1-K(x)}{|x|} \geq \frac{2 m}{N^{*}}$, then there exists at least one minimizer for (1.1) at $N=N^{*}$.

Theorems 1.1 and 1.2 imply that the existence of minimizers depends greatly on the potential $K(x)$ near the origin and near the spatial infinity as well. By applying Ekeland's variational principle (cf. [22, Theorem 5.1]), Theorem 1.2 shows that the existence of minimizers may occur at the critical stellar mass $N=N^{*}$, which depends a little more on the local profile of $K(x)$ near the origin. This is in contrast to the case of the Coulomb-type potential where $K(x) \equiv 1$, for which any minimizer does not exist at $N=N^{*}$ (see [9] and references therein). As a completion of Theorem 1.2, one may wonder whether there is no minimizer for (1.1) at $N=N^{*}$, if $K(x)$ satisfies $0<\liminf _{|x| \rightarrow 0} \frac{1-K(x)}{|x|}<\frac{2 m}{N^{*}}$.

Without loss of generality, one can restrict the minimization of (1.1) to positive functions. Indeed, we note from [19, Theorem 7.12] that $\mathcal{E}(u) \geq \mathcal{E}(|u|)$ for any $u \in H^{\frac{1}{2}}\left(\mathbb{R}^{3}\right)$, which then implies that any minimizer $u_{N}$ of (1.1) satisfies either $u_{N} \geq 0$ or $u_{N} \leq 0$ in $\mathbb{R}^{3}$. Further, $u_{N}$ satisfies the Euler-Lagrange equation

$$
\left[\sqrt{-\Delta+m^{2}}-m+V(x)\right] u_{N}=-\mu_{N} u_{N} \text { in } \mathbb{R}^{3},
$$

where $V(x):=-\left(\frac{K(x)}{|x|} *\left|u_{N}\right|^{2}\right)$ and $\mu_{N} \in \mathbb{R}$ is the associated Lagrange multiplier. If $u_{N} \geq 0$ and $u_{N} \not \equiv 0$ in $\mathbb{R}^{3}$, by (1.12) we then deduce from [19, Theorem 11.8] that $u_{N}>0$ in $\mathbb{R}^{3}$. Therefore, for convenience we next focus on positive minimizers of (1.1).

As discussed in Remark 2.1, the proof of Theorem 1.1 also implies that there is no minimizer for (1.1) at $N=N^{*}$ for the more general case where $K(x)=1-o(|x|) \geq 0$ as $|x| \rightarrow 0$. Inspired by our recent work [12], we next focus on this case to derive the refined behavior of minimizers $u_{N}$ of (1.1) as $N$ approaches the critical value $N^{*}:=\|Q\|_{2}^{2}$ from below.

Theorem 1.3. Suppose $m>0$ and $K(x)$ satisfies (1.9), (1.10) and $K(x)=1-o(|x|) \geq 0$ as $|x| \rightarrow 0$. Let $u_{N}$ be a positive minimizer of (1.1) for $N<N^{*}$. Then for any sequence $\left\{N_{k}\right\}$ with $N_{k} \nearrow N^{*}$ as $k \rightarrow \infty$, there exist a subsequence, still denoted by $\left\{N_{k}\right\}$, of $\left\{N_{k}\right\}$ and $\left\{y_{N_{k}}\right\} \subset \mathbb{R}^{3}$ as well as $y_{0} \in \mathbb{R}^{3}$ such that

$$
\lim _{k \rightarrow \infty} \varepsilon_{N_{k}}^{\frac{3}{2}} u_{N_{k}}\left(\varepsilon_{N_{k}} x+\varepsilon_{N_{k}} y_{N_{k}}\right)=\left(\frac{1}{N^{*}}\right)^{\frac{3}{2}} Q_{0}\left(\frac{\left|x-y_{0}\right|}{N^{*}}\right) \text { strongly in } H^{\frac{1}{2}}\left(\mathbb{R}^{3}\right),
$$

where $Q_{0} \in \mathcal{G}$ is a ground state of (1.4), and $\varepsilon_{N_{k}}>0$ is given by

$$
\varepsilon_{N_{k}}:=\left(\int_{\mathbb{R}^{2}}\left|(-\Delta)^{1 / 4} u_{N_{k}}\right|^{2} d x\right)^{-1} \rightarrow 0 \text { as } k \rightarrow \infty .
$$

Additionally, if $K(x)$ satisfies

1 Private discussions with Robert Seiringer. 


$$
\lim _{|x| \rightarrow 0} \frac{1-K(x)}{|x|^{p}}=\beta \in(0, \infty), \quad 1<p \leq \infty,
$$

then (1.13) holds for $\varepsilon_{N_{k}}>0$ satisfying

$$
\lim _{k \rightarrow \infty} \frac{N^{*} \varepsilon_{N_{k}}}{\left(N^{*}-N_{k}\right)^{\frac{1}{\ell+1}}}=\left\{\begin{array}{lc}
\left(\frac{2}{(p-1) \beta \gamma_{0}}\right)^{\frac{1}{p}}, & 1<p<2 ; \\
\left(\frac{2}{m^{2} \lambda_{0}+\beta \gamma_{0}}\right)^{\frac{1}{2}}, & p=2 ; \\
\left(\frac{2}{m^{2} \lambda_{0}}\right)^{\frac{1}{2}}, & 2<p \leq \infty ;
\end{array}\right.
$$

and the energy $e\left(N_{k}\right)$ satisfies

$$
\lim _{k \rightarrow \infty} \frac{e\left(N_{k}\right)+m N_{k}}{\left(N^{*}-N_{k}\right)^{\frac{\ell}{\ell+1}}}=\frac{\ell+1}{\ell} \ell^{\frac{1}{\ell+1}}\left(\frac{\hat{\kappa}_{p}}{2}\right)^{\frac{1}{\ell+1}},
$$

where $\ell=\min \{2, p\}-1>0$ and

$$
\lambda_{0}:=\int_{\mathbb{R}^{3}} \frac{\left|\widehat{Q}_{0}(\mathrm{k})\right|^{2}}{|\mathrm{k}|} d \mathrm{k} \in(0, \infty) \text { and } \gamma_{0}:=\int_{\mathbb{R}^{3}}\left(|x|^{p-1} *\left|Q_{0}\right|^{2}\right)\left|Q_{0}\right|^{2} d x \in(0, \infty],
$$

and $\hat{\kappa}_{p}>0$ satisfies

$$
\hat{\kappa}_{p}=\left\{\begin{array}{lr}
\beta \gamma_{0}, & 1<p<2 ; \\
\beta \gamma_{0}+m^{2} \lambda_{0}, & p=2 ; \\
m^{2} \lambda_{0}, & 2<p \leq \infty .
\end{array}\right.
$$

As a byproduct, Theorem 1.3 also presents a refined energy estimate (1.17) of $e(N)$ as $N \nearrow N^{*}$. The proof of Theorem 1.3 shows that the polynomial decaying characters (1.7) and (1.8) of $Q_{0} \in \mathcal{G}$ result in more delicate energy estimates and more complicated blow up analysis of minimizers as $N \nearrow N^{*}$, compared with those obtained in [12-14] for attractive BEC. Also, one may use (1.7) and (1.8) to check that the constant $\gamma_{0}$ in (1.18) satisfies $\gamma_{0}<\infty$ for $1<p<6$ (see also (3.21) for details), and therefore, the constant $\hat{\kappa}_{p}$ in (1.19) is always finite. Finally, denote

$$
\eta:=\left(\frac{m^{2}}{2} \int_{\mathbb{R}^{3}} \frac{\left|\widehat{Q}_{0}(k)\right|^{2}}{|k|} d k\right)^{-\frac{1}{2}}
$$

where $Q_{0} \in \mathcal{G}$ is a ground state of (1.4). Then for the case where $K(x) \equiv 1$, Theorem 1.3 with $p=\infty$ is reduced immediately to the following simplified one.

Proposition 1.4. Suppose $m>0, K(x) \equiv 1$ and let $u_{N}$ be a positive minimizer of (1.1) for $N<N^{*}$. Then for any sequence $\left\{N_{k}\right\}$ with $N_{k} \nearrow N^{*}$ as $k \rightarrow \infty$, there exist a subsequence, still denoted by $\left\{N_{k}\right\}$, of $\left\{N_{k}\right\}$ and $\left\{y_{N_{k}}\right\} \subset \mathbb{R}^{3}$ as well as $y_{0} \in \mathbb{R}^{3}$ such that

$$
\lim _{k \rightarrow \infty}\left(N^{*}-N_{k}\right)^{\frac{3}{2}} u_{N_{k}}\left[\left(N^{*}-N_{k}\right)^{\frac{1}{2}}\left(x+y_{N_{k}}\right)\right]=\eta^{\frac{3}{2}} Q_{0}\left(\eta\left|x-y_{0}\right|\right) \text { strongly in } H^{\frac{1}{2}}\left(\mathbb{R}^{3}\right) .
$$

Moreover, the energy e $\left(N_{k}\right)$ satisfies

$$
\lim _{k \rightarrow \infty} \frac{e\left(N_{k}\right)+m N_{k}}{\left(N^{*}-N_{k}\right)^{\frac{1}{2}}}=\frac{2}{\eta} .
$$

After this paper was submitted, we learned that a similar minimization problem of (1.1), where a trapping potential $V(x)$ is imposed, was also studied in [23]. This paper is organized as follows. Section 2 is devoted to the proof of Theorems 1.1 and 1.2, and particularly we shall employ in Subsection 2.1 Ekeland's variational principle to establish the existence of minimizers at $N=N^{*}$ as stated in Theorem 1.2. In Section 3 we then complete the proof of Theorem 1.3 on the mass concentration of minimizers as $N \nearrow N^{*}$. We shall always assume $m>0$ throughout the paper. 


\section{Existence and nonexistence of minimizers}

The main purpose of this section is to establish Theorems 1.1 and 1.2 on the existence and nonexistence of minimizers. Towards this aim, we first address the following energy estimate of (1.1).

Lemma 2.1. Suppose that either $m>0$ is large sufficiently or $K(x) \geq O\left(|x|^{-\alpha}\right)$ as $|x| \rightarrow \infty$ with $0<\alpha<1$. Then we have $e(N)<0$ for any $N>0$.

Proof. For $m>0$, using the operator inequality,

$$
2 m \sqrt{-\Delta+m^{2}} \leq-\Delta+2 m^{2},
$$

we have for any $N>0$,

$$
e(N) \leq e^{c}(N):=\inf \left\{\mathcal{E}^{c}(\psi): \psi \in H^{1}\left(\mathbb{R}^{3}\right), \int_{\mathbb{R}^{3}}|\psi(x)|^{2} d x=N\right\},
$$

where the energy functional $\mathcal{E}^{c}(\psi)$ is of the form

$$
\mathcal{E}^{c}(\psi)=\frac{1}{2 m} \int_{\mathbb{R}^{3}}|\nabla \psi|^{2} d x-\frac{1}{2} \int_{\mathbb{R}^{3}}\left(\frac{K(x)}{|x|} *|\psi|^{2}\right)|\psi|^{2} d x .
$$

For any $N>0$, if one chooses $\psi(x) \in C_{c}^{\infty}\left(\mathbb{R}^{3}\right)$, such that $\int_{\mathbb{R}^{3}}|\psi|^{2} d x=N$, and sets

$$
\psi_{\lambda}:=\lambda^{\frac{3}{2}} \psi(\lambda x), \lambda>0,
$$

then

$$
\int_{\mathbb{R}^{3}}\left|\psi_{\lambda}\right|^{2} d x=N
$$

and

$$
\mathcal{E}^{c}\left(\psi_{\lambda}\right)=\frac{\lambda^{2}}{2 m} \int_{\mathbb{R}^{3}}|\nabla \psi|^{2} d x-\frac{\lambda}{2} \int_{\mathbb{R}^{3}}\left(\frac{K(x / \lambda)}{|x|} *|\psi|^{2}\right)|\psi|^{2} d x .
$$

Inspired by $[18,19]$, we shall carry out the proof by discussing separately the following two cases:

(1). Suppose that $m>0$ is large sufficiently. In this case, by taking $\lambda=1$ and $\psi_{1}>0$ is a suitable test function as above, we obtain from (2.1) and (2.2) that

$$
e(N) \leq e^{c}(N) \leq \mathcal{E}^{c}\left(\psi_{1}\right)<0 \text { for all } N>0,
$$

and we are done.

(2). Suppose that $K(x) \geq O\left(|x|^{-\alpha}\right)$ as $|x| \rightarrow \infty$ with $0<\alpha<1$. In this case, we further deduce that

$$
\begin{aligned}
& \int_{\mathbb{R}^{3}}\left(\frac{K(x / \lambda)}{|x|} *|\psi|^{2}\right)|\psi|^{2} d x \\
& \quad \geq \int_{|x|<R} \int_{1<|y|<R} \frac{K(y / \lambda)}{|y|} \psi^{2}(x-y) \psi^{2}(x) d x d y \\
& \quad \geq C \lambda^{\alpha} \int_{|x|<R} \int_{1<|y|<R} \frac{K_{\infty}}{|y|^{1+\alpha}} \psi^{2}(x-y) \psi^{2}(x) d x d y \geq C \lambda^{\alpha}>0 \text { as } \lambda \rightarrow 0 .
\end{aligned}
$$

Applying (2.2), we then have 


$$
\mathcal{E}^{c}\left(\psi_{\lambda}\right)<0 \text { if } \lambda>0 \text { is small, }
$$

and further,

$$
e(N) \leq e^{c}(N) \leq \mathcal{E}^{c}\left(\psi_{\lambda}\right)<0 \text { for all } N>0,
$$

which completes the proof of Lemma 2.1 .

The conclusion $e(N)<0$ of Lemma 2.1 gives essentially the strict subadditivity condition of $e(N)$, based on which we shall be able to apply the concentration-compactness-type method (cf. $[9,21]$ ) to deriving the existence of minimizers. More precisely, we shall follow Lemma 2.1 to establish the following theorem, which then gives Theorem 1.1.

Theorem 2.2. Suppose $m>0$ and $K(x)$ satisfies (1.9). Then we have

1. If $0 \leq N<N^{*}:=\|Q\|_{2}^{2}$ and $K(x)$ also satisfies (1.10), there exists at least one minimizer for (1.1).

2. If $N>N^{*}$, there is no minimizer for (1.1).

3. If $K(x)$ satisfies additionally that $K(x)=1-o(|x|) \geq 0$ as $|x| \rightarrow 0$, then there is no minimizer for (1.1) at $N=N^{*}$.

Proof. Inspired by [9, Theorem 2.1], [12, Theorem 1] and [20, Theorem 4], we divide our proof into three parts.

(i). Under the additional assumption (1.10), we first prove that (1.1) has at least one minimizer for all $0<N<N^{*}$. Actually, if $u \in H^{\frac{1}{2}}\left(\mathbb{R}^{3}\right)$ and $\|u\|_{2}^{2}=N$, we then deduce from the inequality (1.3) that

$$
\begin{aligned}
\mathcal{E}(u) & \geq\left\|\left(-\Delta+m^{2}\right)^{1 / 4} u\right\|_{2}^{2}-\frac{1}{2} \int_{\mathbb{R}^{3}}\left(\frac{1}{|x|} *|u|^{2}\right)(x)|u|^{2} d x-m N \\
& \geq\left\|(-\Delta)^{1 / 4} u\right\|_{2}^{2}-\frac{1}{N^{*}}\left\|(-\Delta)^{1 / 4} u\right\|_{2}^{2}\|u\|_{2}^{2}-m N \\
& =\frac{N^{*}-N}{N^{*}}\left\|(-\Delta)^{1 / 4} u\right\|_{2}^{2}-m N,
\end{aligned}
$$

which implies that $\mathcal{E}(u)$ is bounded uniformly from below for all $0<N<N^{*}$. Choose a minimizing sequence $\left\{u_{n}\right\} \subset H^{\frac{1}{2}}\left(\mathbb{R}^{3}\right)$ satisfying $\left\|u_{n}\right\|_{2}^{2}=N$ and $\lim _{n \rightarrow \infty} \mathcal{E}\left(u_{n}\right)=e(N)$. It follows from (2.4) that the sequence $\left\{u_{n}\right\}$ is bounded uniformly in $H^{\frac{1}{2}}\left(\mathbb{R}^{3}\right)$. Furthermore, similar to the proof of [9, Theorem 2.1], applying Lemma 2.1 and the concentration-compactness-type method (cf. $[9,21]$ ), one can derive that there exist subsequences $\left\{u_{n_{k}}\right\}$ and $\left\{y_{k}\right\} \subset \mathbb{R}^{3}$ such that the sequence

$$
\tilde{u}_{k}(\cdot):=u_{n_{k}}\left(\cdot+y_{k}\right)
$$

satisfies

$$
\tilde{u}_{k} \rightarrow u \text { strongly in } H^{\frac{1}{2}}\left(\mathbb{R}^{3}\right) \text { as } k \rightarrow \infty,
$$

and

$$
\lim _{k \rightarrow \infty} \int_{\mathbb{R}^{3}}\left(\frac{K(x)}{|x|} *\left|\tilde{u}_{k}\right|^{2}\right)(x)\left|\tilde{u}_{k}(x)\right|^{2} d x=\int_{\mathbb{R}^{3}}\left(\frac{K(x)}{|x|} *|u|^{2}\right)(x)|u(x)|^{2} d x
$$

for some $u \in H^{\frac{1}{2}}\left(\mathbb{R}^{3}\right)$. We therefore conclude that $\int_{\mathbb{R}^{3}}|u(x)|^{2} d x=N$ and $\mathcal{E}(u)=e(N)$ by the weak lower semicontinuity. This implies the existence of minimizers for all $0<N<N^{*}$.

(ii). We next show that there is no minimizer for (1.1) as soon as $N>N^{*}$. Choose any $Q \in \mathcal{G}$, and define

$$
u_{\tau}(x)=\frac{\sqrt{N} \tau^{\frac{3}{2}}}{\|Q\|_{2}} Q(\tau x),
$$

so that $\int_{\mathbb{R}^{3}} u_{\tau}^{2}(x) d x=N$. Using the inequality that $\sqrt{-\Delta+m^{2}}-m \leq \sqrt{-\Delta}$, we then obtain from (1.6) that 


$$
\begin{aligned}
e(N) \leq \mathcal{E}\left(u_{\tau}\right) \leq & \left\|(-\Delta)^{1 / 4} u_{\tau}\right\|_{2}^{2}-\frac{1}{2} \int_{\mathbb{R}^{3}}\left(\frac{K(x)}{|x|} *\left|u_{\tau}\right|^{2}\right)(x)\left|u_{\tau}\right|^{2} d x \\
= & \frac{N \tau}{N^{*}}\left\|(-\Delta)^{1 / 4} Q\right\|_{2}^{2}-\frac{N^{2} \tau}{2\left(N^{*}\right)^{2}} \int_{\mathbb{R}^{3}}\left(\frac{K\left(\frac{x}{\tau}\right)}{|x|} * Q^{2}\right)(x) Q^{2}(x) d x \\
= & \frac{N \tau}{2 N^{*}}\left[\left(1-\frac{N}{N^{*}}\right) \int \frac{1}{\mathbb{R}^{3}} * Q^{2}\right)(x) Q^{2}(x) d x \\
& \left.+\frac{N}{N^{*}} \int_{\mathbb{R}^{3}}\left(\frac{1-K\left(\frac{x}{\tau}\right)}{|x|} * Q^{2}\right)(x) Q^{2}(x) d x\right] \\
= & \left.\frac{N \tau}{N^{*}}\left[\left(N^{*}-N\right)+\frac{N}{2 N^{*}} \int \frac{1-K\left(\frac{x}{\tau}\right)}{|x|} * Q_{\mathbb{R}^{3}}\right)(x) Q^{2}(x) d x\right] .
\end{aligned}
$$

Since $K(0)=1$, one can check that

$$
\int_{\mathbb{R}^{3}}\left(\frac{1-K\left(\frac{x}{\tau}\right)}{|x|} * Q^{2}\right)(x) Q^{2}(x) d x \rightarrow 0 \text { as } \tau \rightarrow \infty .
$$

It then follows from (2.6) that

$$
e(N) \leq \mathcal{E}\left(u_{\tau}\right) \leq \frac{N \tau}{N^{*}}\left[\left(N^{*}-N\right)+o(1)\right] \text { as } \tau \rightarrow \infty,
$$

which thus yields that $e(N)=-\infty$ if $N>N^{*}$. This implies the non-existence of minimizers as soon as $N>N^{*}$.

(iii). We finally deal with the case $N=N^{*}$ under the additional assumption that $1-K(x)=o(|x|)$ as $|x| \rightarrow 0$. In this case, we use the same trial function $u_{\tau}$ as that of (2.5). It then follows from (1.3) that

$$
\begin{aligned}
-m N^{*} \leq e\left(N^{*}\right) \leq \mathcal{E}\left(u_{\tau}\right) \leq & \left\langle u_{\tau},\left(\sqrt{-\Delta+m^{2}}-\sqrt{-\Delta}\right) u_{\tau}\right\rangle-m N^{*} \\
& +\frac{1}{2} \int_{\mathbb{R}^{3}}\left(\frac{1-K(x)}{|x|} *\left|u_{\tau}\right|^{2}\right)(x)\left|u_{\tau}\right|^{2} d x .
\end{aligned}
$$

Applying Plancherel's theorem, we use the dominated convergence to derive that

$$
\begin{aligned}
& \left\langle u_{\tau},\left(\sqrt{-\Delta+m^{2}}-\sqrt{-\Delta}\right) u_{\tau}\right\rangle \\
& \quad=\int_{\mathbb{R}^{3}}|\widehat{Q}(k)|^{2}\left(\sqrt{\tau^{2} k^{2}+m^{2}}-\tau|k|\right) d k \rightarrow 0 \text { as } \tau \rightarrow \infty .
\end{aligned}
$$

Moreover, since $1-K(x)=o(|x|)$ as $|x| \rightarrow 0$, we also have

$$
\begin{aligned}
& \frac{1}{2} \int_{\mathbb{R}^{3}}\left(\frac{1-K(x)}{|x|} *\left|u_{\tau}\right|^{2}\right)(x)\left|u_{\tau}(x)\right|^{2} d x \\
& \quad=\frac{1}{2} \int_{\mathbb{R}^{3}}\left(\frac{1-K\left(\frac{x}{\tau}\right)}{\left|\frac{x}{\tau}\right|} * Q^{2}\right)(x) Q^{2}(x) d x \rightarrow 0 \text { as } \tau \rightarrow \infty .
\end{aligned}
$$

We now conclude from (2.8)-(2.10) that $e\left(N^{*}\right)=-m N^{*}$ by letting $\tau \rightarrow \infty$. This implies that if there exists a minimizer $u$ for $e\left(N^{*}\right)$, we then have

$$
\begin{aligned}
\left\|\left(-\Delta+m^{2}\right)^{1 / 4} u\right\|_{2}^{2} & =\frac{1}{2} \int_{\mathbb{R}^{3}}\left(\frac{K(x)}{|x|} *|u|^{2}\right)(x)|u(x)|^{2} d x \\
& \leq \frac{1}{2} \int_{\mathbb{R}^{3}}\left(\frac{1}{|x|} *|u|^{2}\right)(x)|u|^{2} d x \leq\left\|(-\Delta)^{1 / 4} u(x)\right\|_{2}^{2},
\end{aligned}
$$


which however contradicts the fact that

$$
\left\|\left(-\Delta+m^{2}\right)^{1 / 4} u\right\|_{2}^{2}>\left\|(-\Delta)^{1 / 4} u\right\|_{2}^{2}
$$

holds for any $m>0$. This proves the nonexistence of minimizers for (1.1) at $N=N^{*}$, which therefore completes the proof of Theorem 2.2.

Remark 2.1. If $K(x) \in C\left(\mathbb{R}^{3}\right)$ satisfies (1.9) and (1.10), we then conclude from Lemma 2.1 and the proof of Theorem 1.1 that the Hartree energy $e(N)$ satisfies

$$
-m N<e(N)<0 \text { for } 0<N<N^{*} \text {, and } e(N)=-\infty \text { for } N>N^{*} .
$$

One can also check that $e(N)$ is strictly decreasing and concave downward for all $0<N<N^{*}$. Additionally, if $K(x)=1-o(|x|) \geq 0$ as $|x| \rightarrow 0$, we then have

$$
\lim _{N \nearrow N^{*}} e(N)=-m N^{*} \text {. }
$$

All these properties are often used in next section.

\subsection{Existence of minimizers at $N=N^{*}$}

One can note that Theorem 1.1 and $(a)$ of Theorem 1.2 follow from Theorem 2.2. To complete the proof of Theorems 1.2, the rest is to derive the following existence of minimizers at $N=N^{*}$.

Theorem 2.3. Suppose $m>0, K(x)$ satisfies (1.9) and (1.10). If $K(x)$ also satisfies either

$$
K(x)=1-O\left(|x|^{p}\right) \geq 0 \text { as }|x| \rightarrow 0 \text { for some constant } 0<p<1,
$$

or

$$
\liminf _{|x| \rightarrow 0} \frac{1-K(x)}{|x|} \geq \frac{2 m}{N^{*}},
$$

then there exists at least one minimizer for (1.1) at $N=N^{*}$.

This subsection is devoted to the proof of Theorem 2.3. Under the assumptions of Theorem 2.3, consider the manifold

$$
\mathcal{A}:=\left\{u: u \in H^{\frac{1}{2}}\left(\mathbb{R}^{3}\right) \text { and } \int_{\mathbb{R}^{3}}|u|^{2} d x=N^{*}\right\},
$$

and define

$$
d(u, v)=\|u-v\|_{H^{\frac{1}{2}}}, u, v \in \mathcal{A},
$$

so that $(\mathcal{A}, d)$ is a complete metric space. By Ekeland's variational principle (cf. [22, Theorem 5.1]), then there exists a minimizing sequence $\left\{u_{n}\right\}$ of $e\left(N^{*}\right)$ such that

$$
\begin{aligned}
& e\left(N^{*}\right) \leq \mathcal{E}\left(u_{n}\right) \leq e\left(N^{*}\right)+\frac{1}{n}, \\
& \mathcal{E}(v) \geq \mathcal{E}\left(u_{n}\right)-\frac{1}{n}\left\|u_{n}-v\right\|_{H^{\frac{1}{2}}} \text { for any } v \in \mathcal{A} .
\end{aligned}
$$

By applying (2.15) and (2.16), we shall derive the claim that

$$
\left\{u_{n}\right\} \text { is bounded uniformly in } \mathcal{A} \text {. }
$$

Note from the assumption (1.10) that Lemma 2.1 holds. Thus, if the claim (2.17) holds, by applying Lemma 2.1, the same argument of (i) in the proof of Theorem 2.2 then gives the existence of minimizers at $N=N^{*}$, and Theorem 2.3 is therefore proved. 
The rest part of this subsection is to derive the claim (2.17). On the contrary, we now suppose that (2.17) is false, i.e. $\left\{u_{n}\right\}$ is unbounded in $\mathcal{A}$, and we shall finally derive a contradiction. In this case, then there exists a subsequence of $\left\{u_{n}\right\}$, still denoted by $\left\{u_{n}\right\}$, such that $\left\|u_{n}\right\|_{H^{\frac{1}{2}}} \rightarrow \infty$ as $n \rightarrow \infty$. Note from (1.3) and (2.15) that for $m>0$,

$$
0 \leq \int_{\mathbb{R}^{3}}\left|(-\Delta)^{1 / 4} u_{n}(x)\right|^{2} d x-\frac{1}{2} \int_{\mathbb{R}^{3}}\left(\frac{K(x)}{|x|} *\left|u_{n}\right|^{2}\right)\left|u_{n}(x)\right|^{2} d x \leq e\left(N^{*}\right)+m N^{*}+\frac{1}{n},
$$

which then implies that

$$
\begin{gathered}
\int_{\mathbb{R}^{3}}\left|(-\Delta)^{1 / 4} u_{n}(x)\right|^{2} d x \rightarrow \infty \text { as } n \rightarrow \infty, \\
\frac{1}{2} \int_{\mathbb{R}^{3}}\left(\frac{K(x)}{|x|} *\left|u_{n}\right|^{2}\right)\left|u_{n}(x)\right|^{2} d x \rightarrow \infty \text { as } n \rightarrow \infty .
\end{gathered}
$$

Define now

$$
\epsilon_{n}^{-1}:=\int_{\mathbb{R}^{3}}\left(\frac{K(x)}{|x|} *\left|u_{n}\right|^{2}\right)\left|u_{n}(x)\right|^{2} d x, \epsilon_{n}>0,
$$

so that $\epsilon_{n} \rightarrow 0$ as $n \rightarrow \infty$ in view of (2.19). It then follows from (2.18) that there exists a constant $M>0$, independent of $n$, such that

$$
0<M \epsilon_{n}^{-1} \leq \int_{\mathbb{R}^{3}}\left|(-\Delta)^{1 / 4} u_{n}(x)\right|^{2} d x \leq \frac{1}{M} \epsilon_{n}^{-1}+e\left(N^{*}\right)+m N^{*} \text { as } n \rightarrow \infty .
$$

In view of above facts, we next define the $L^{2}\left(\mathbb{R}^{2}\right)$-normalized function

$$
\tilde{w}_{n}(x):=\epsilon_{n}^{\frac{3}{2}} u_{n}\left(\epsilon_{n} x\right) \text {. }
$$

It then yields from (2.20) and (2.21) that

$$
\begin{gathered}
\int_{\mathbb{R}^{3}}\left(\frac{K\left(\epsilon_{n} x\right)}{|x|} *\left|\tilde{w}_{n}\right|^{2}\right)\left|\tilde{w}_{n}(x)\right|^{2} d x=1, \\
M \leq \int_{\mathbb{R}^{3}}\left|(-\Delta)^{1 / 4} \tilde{w}_{n}(x)\right|^{2} d x \leq \frac{1}{M}+\epsilon_{n}\left[e\left(N^{*}\right)+m N^{*}\right],
\end{gathered}
$$

where $M>0$, independent of $n$, is the same as that in (2.21).

We claim that there exist a sequence $\left\{y_{\epsilon_{n}}\right\}$ and positive constants $R$ and $\eta$ such that

$$
\liminf _{\epsilon_{n} \rightarrow 0} \int_{B_{R}\left(y_{\epsilon_{n}}\right)}\left|\tilde{w}_{n}(x)\right|^{2} d x \geq \eta>0 .
$$

On the contrary, suppose that (2.24) is false. A proof similar to that of [9, Lemma A.2] then gives that

$$
\int_{\mathbb{R}^{3}}\left(\frac{K\left(\epsilon_{n} x\right)}{|x|} *\left|\tilde{w}_{n}\right|^{2}\right)(x)\left|\tilde{w}_{n}(x)\right|^{2} d x \rightarrow 0 \text { as } n \rightarrow \infty,
$$

which however contradicts (2.23). Therefore, the above claim (2.24) holds. For the sequence $\left\{y_{\epsilon_{n}}\right\}$ obtained in (2.24), we next set

$$
w_{n}(x)=\tilde{w}_{n}\left(x+y_{\epsilon_{n}}\right)=\epsilon_{n}^{\frac{3}{2}} u_{n}\left(\epsilon_{n} x+\epsilon_{n} y_{\epsilon_{n}}\right) .
$$

Note from (2.23) that $w_{n} \in H^{\frac{1}{2}}\left(\mathbb{R}^{3}\right)$, and the estimate (2.24) implies that 


$$
\liminf _{\epsilon_{n} \rightarrow 0} \int_{B_{R}(0)}\left|w_{n}(x)\right|^{2} d x \geq \eta>0,
$$

where positive constants $R$ and $\eta$ are given by (2.24). In the following we need to establish the following crucial lemma.

Lemma 2.4. Under the assumptions of Theorem 2.3, let $w_{n} \in H^{\frac{1}{2}}\left(\mathbb{R}^{3}\right)$ be defined by (2.25). Then $w_{n} \rightarrow w_{0}$ strongly in $L^{p}\left(\mathbb{R}^{3}\right)$ for all $p \in[2,3)$, where $w_{0}$ is a nonzero solution of the problem

$$
\sqrt{-\Delta} w(x)+\frac{1}{N^{*}} w(x)-\left(\frac{1}{|x|} *|w|^{2}\right) w(x)=0 \text { in } \mathbb{R}^{3} .
$$

Proof. For any $\varphi(x) \in C_{0}^{\infty}\left(\mathbb{R}^{2}\right)$, define

$$
\tilde{\varphi}(x)=\epsilon_{n}^{-\frac{1}{2}} \varphi\left(\frac{x-\epsilon_{n} y_{\epsilon_{n}}}{\epsilon_{n}}\right), \quad j(\tau, \sigma)=\frac{1}{2} \int_{\mathbb{R}^{3}}\left|u_{n}+\tau u_{n}+\sigma \tilde{\varphi}\right|^{2} d x-\frac{N^{*}}{2} .
$$

Then $j(\tau, \sigma)$ satisfies

$$
j(0,0)=0, \quad \frac{\partial j(0,0)}{\partial \tau}=\int_{\mathbb{R}^{3}}\left|u_{n}(x)\right|^{2} d x=N^{*} \quad \text { and } \quad \frac{\partial j(0,0)}{\partial \sigma}=\int_{\mathbb{R}^{3}} u_{n}(x) \tilde{\varphi}(x) d x .
$$

Applying the implicit function theorem, we thus obtain that there exist a constant $\delta_{n}>0$ and a function $\tau(\sigma) \in$ $C^{1}\left(\left(-\delta_{n}, \delta_{n}\right), \mathbb{R}\right)$, where $|\sigma|>0$ is sufficiently small, such that

$$
\tau(0)=0, \quad \tau^{\prime}(0)=-\frac{1}{N^{*}} \int_{\mathbb{R}^{3}} u_{n}(x) \tilde{\varphi}(x) d x, \quad \text { and } \quad j(\tau(\sigma), \sigma)=0 .
$$

Therefore, we have $u_{n}+\tau(\sigma) u_{n}+\sigma \tilde{\varphi} \in \mathcal{A}$, where $\sigma \in\left(-\delta_{n}, \delta_{n}\right)$. By applying (2.16), we get that

$$
\mathcal{E}\left(u_{n}+\tau(\sigma) u_{n}+\sigma \tilde{\varphi}\right)-\mathcal{E}\left(u_{n}\right) \geq-\frac{1}{n}\left\|\tau(\sigma) u_{n}+\sigma \tilde{\varphi}\right\|_{H^{\frac{1}{2}}} .
$$

Taking the limits $\sigma \rightarrow 0^{+}$and $\sigma \rightarrow 0^{-}$, respectively, we then deduce that

$$
\left|\left\langle\mathcal{E}^{\prime}\left(u_{n}\right), \tau^{\prime}(0) u_{n}+\tilde{\varphi}\right\rangle\right| \leq \frac{1}{n}\left\|\tau^{\prime}(0) u_{n}+\tilde{\varphi}\right\|_{H^{\frac{1}{2}}} .
$$

On the other hand, the definition of (2.25) gives that

$$
\begin{aligned}
\frac{1}{2}\left\langle\mathcal{E}^{\prime}\left(u_{n}\right), \tilde{\varphi}\right\rangle= & \int_{\mathbb{R}^{3}} \varphi(x)\left(\sqrt{-\Delta+m^{2} \epsilon_{n}^{2}}-m \epsilon_{n}\right) w_{n}(x) d x \\
& -\int_{\mathbb{R}^{3}}\left(\frac{K\left(\epsilon_{n} x\right)}{|x|} *\left|w_{n}\right|^{2}\right) w_{n}(x) \varphi(x) d x .
\end{aligned}
$$

By setting

$$
\mu_{n}:=\left\langle\mathcal{E}^{\prime}\left(u_{n}\right), u_{n}\right\rangle,
$$

we then deduce from (2.29) that

$$
\begin{aligned}
& \left\|\tau^{\prime}(0) u_{n}+\tilde{\varphi}\right\|_{H^{\frac{1}{2}}}<C \epsilon_{n}^{\frac{1}{2}}, \\
& \tau^{\prime}(0)=-\frac{1}{N^{*}} \int_{\mathbb{R}^{3}} u_{n}(x) \tilde{\varphi}(x) d x=-\frac{\epsilon_{n}}{N^{*}} \int_{\mathbb{R}^{3}} w_{n}(x) \varphi(x) d x, \\
& \left|\mu_{n} \epsilon_{n}+1\right|=\left.\left|\mu_{n} \epsilon_{n}+\epsilon_{n} \int_{\mathbb{R}^{3}}\left(\frac{K(x)}{|x|} *\left|u_{n}\right|^{2}\right)\right| u_{n}(x)\right|^{2} d x \mid \rightarrow 0 \text { as } n \rightarrow \infty .
\end{aligned}
$$


Thus, the estimates (2.28)-(2.30) yield that

$$
\begin{gathered}
\mid \int_{\mathbb{R}^{3}} \varphi(x)\left(\sqrt{-\Delta+m^{2} \epsilon_{n}^{2}}-m \epsilon_{n}\right) w_{n}(x) d x-\frac{\mu_{n} \epsilon_{n}}{N^{*}} \int_{\mathbb{R}^{3}} w_{n}(x) \varphi(x) d x \\
\quad-\int_{\mathbb{R}^{3}}\left(\frac{K\left(\epsilon_{n} x\right)}{|x|} *\left|w_{n}\right|^{2}\right) w_{n}(x) \varphi(x) d x \mid \leq \frac{C \epsilon_{n}^{\frac{1}{2}}}{n} .
\end{gathered}
$$

By the estimate (2.26), we thus derive from (2.31) that $w_{n} \rightarrow w_{0} \not \equiv 0$ in $H^{1}\left(\mathbb{R}^{2}\right)$ as $n \rightarrow \infty$, where $w_{0}$ satisfies the equation (2.27), due to the assumption that $K(0)=1$. Further, we deduce from (2.26) that

$$
0<\int_{\mathbb{R}^{3}}\left|w_{0}\right|^{2} d x \leq \liminf _{n \rightarrow \infty} \int_{\mathbb{R}^{3}}\left|w_{n}\right|^{2} d x=N^{*} .
$$

Moreover, it follows from (2.27) and the standard Pohozaev identity that

$$
\frac{1}{N^{*}} \int_{\mathbb{R}^{3}}\left|w_{0}\right|^{2} d x=\int_{\mathbb{R}^{3}}|k|\left|\widehat{w}_{0}\right|^{2} d k=\frac{1}{2} \int_{\mathbb{R}^{3}}\left(\frac{1}{|x|} *\left|w_{0}\right|^{2}\right)\left|w_{0}\right|^{2} d x .
$$

We thus derive from (1.3) that

$$
\frac{N^{*}}{2} \leq \frac{\int_{\mathbb{R}^{3}}|k|\left|\widehat{w}_{0}\right|^{2} d k \cdot \int_{\mathbb{R}^{3}}\left|w_{0}\right|^{2} d x}{\int_{\mathbb{R}^{3}}\left(\frac{1}{|x|} *\left|w_{0}\right|^{2}\right)\left|w_{0}\right|^{2} d x}=\frac{\int_{\mathbb{R}^{3}}\left|w_{0}\right|^{2} d x}{2} .
$$

This together with (2.32) indicates that

$$
w_{n} \rightarrow w_{0} \text { strongly in } L^{p}\left(\mathbb{R}^{3}\right),
$$

for all $p \in[2,3)$ in view of $H^{\frac{1}{2}}\left(\mathbb{R}^{3}\right)$ boundness, and the proof is complete.

Completion for the Proof of (2.17). Case (I): If $K(x)$ satisfies (2.13). By applying Fatou's lemma, we obtain from Lemma 2.4 that for the constant $R>0$ given in (2.26), there exists a constant $C=C(R)>0$ such that

$$
\begin{aligned}
& \lim _{n \rightarrow \infty} \frac{1}{2 \epsilon_{n}^{p}} \int_{B_{R}(0)} \int_{B_{R}(0)} \frac{1-K\left(\epsilon_{n} x-\epsilon_{n} y\right)}{|x-y|}\left|w_{n}(x)\right|^{2}\left|w_{n}(y)\right|^{2} d y d x \\
& \geq C \lim _{n \rightarrow \infty} \int_{B_{R}(0)} \int_{B_{R}(0)} \frac{\left|w_{n}(x)\right|^{2}\left|w_{n}(y)\right|^{2}}{|x-y|^{1-p}} d y d x \\
& \geq C \int_{B_{R}(0)} \int_{B_{R}(0)} \lim _{n \rightarrow \infty} \frac{\left|w_{n}(x)\right|^{2}\left|w_{n}(y)\right|^{2}}{(|x|+|y|)^{1-p}} d y d x \geq C(R) .
\end{aligned}
$$

Since $0<p<1$, we then derive from (2.34) that

$$
\lim _{n \rightarrow \infty} \frac{e\left(N^{*}\right)+m N^{*}}{\epsilon_{n}^{p-1}} \geq \lim _{n \rightarrow \infty} \frac{1}{2 \epsilon_{n}^{p}} \int_{\mathbb{R}^{3}}\left(\frac{1-K\left(\epsilon_{n} x\right)}{|x|} *\left|w_{n}\right|^{2}\right)\left|w_{n}(x)\right|^{2} d x \geq C(R)>0,
$$

which is impossible, and (2.17) is therefore proved. 
Case (II): If $K(x)$ satisfies (2.14). By using Fatou's lemma again, in this case we deduce from (2.33) that

$$
\begin{aligned}
& \liminf _{n \rightarrow \infty} \int_{\mathbb{R}^{3}}\left(\frac{1-K(x)}{|x|} *\left|u_{n}\right|^{2}\right)\left|u_{n}(x)\right|^{2} d x \\
& \quad=\liminf _{n \rightarrow \infty} \int_{\mathbb{R}^{3}}\left(\frac{1-K\left(\epsilon_{n} x\right)}{\left|\epsilon_{n} x\right|} *\left|w_{n}\right|^{2}\right)\left|w_{n}(x)\right|^{2} d x \\
& \geq \int_{\mathbb{R}^{3}} \liminf _{n \rightarrow \infty}\left(\frac{1-K\left(\epsilon_{n} x\right)}{\left|\epsilon_{n} x\right|} *\left|w_{n}\right|^{2}\right)\left|w_{n}(x)\right|^{2} d x \geq \frac{2 m}{N^{*}}\left(N^{*}\right)^{2}=2 m N^{*} .
\end{aligned}
$$

Applying (1.3), the above estimate then yields that

$$
\begin{aligned}
e\left(N^{*}\right)= & \lim _{n \rightarrow \infty} \mathcal{E}\left(u_{n}\right) \\
= & -m N^{*}+\lim _{n \rightarrow \infty}\left\{\left\langle u_{n}, \sqrt{-\Delta} u_{n}\right\rangle-\frac{1}{2} \int_{\mathbb{R}^{3}}\left(\frac{1}{|x|} *\left|u_{N}\right|^{2}\right)(x)\left|u_{n}\right|^{2} d x\right. \\
& \left.+\left\langle u_{n},\left(\sqrt{-\Delta+m^{2}}-\sqrt{-\Delta}\right) u_{n}\right\rangle+\frac{1}{2} \int_{\mathbb{R}^{3}}\left(\frac{1-K(x)}{|x|} *\left|u_{n}\right|^{2}\right)\left|u_{n}\right|^{2} d x\right\} \\
\geq & -m N^{*}+\frac{1}{2} \liminf _{n \rightarrow \infty} \int_{\mathbb{R}^{3}}\left(\frac{1-K(x)}{|x|} *\left|u_{n}\right|^{2}\right)\left|u_{n}\right|^{2} d x \geq 0,
\end{aligned}
$$

which however contradicts Lemma 2.1 under the assumption (1.10). This verifies that (2.17) also holds, and the proof is done.

\section{Mass concentration as $N \nearrow N^{*}$}

This section is focused on the proof of Theorem 1.3, for which we always assume that $K(x)$ satisfies (1.9), (1.10) and $K(x)=1-o(|x|) \geq 0$ as $|x| \rightarrow 0$. Denote $u_{N}$ a positive minimizer of (1.1) with $0<N<N^{*}$, so that $u_{N}$ satisfies the Euler-Lagrange equation

$$
\left(\sqrt{-\Delta+m^{2}}-m\right) u_{N}-\left(\frac{K(x)}{|x|} *\left|u_{N}\right|^{2}\right) u_{N}=-\mu_{N} u_{N} \text { in } \mathbb{R}^{3},
$$

where the Lagrange multiplier $\mu_{N} \in \mathbb{R}$ satisfies

$$
N \mu_{N}=-2 e(N)+\left\langle u_{N},\left(\sqrt{-\Delta+m^{2}}-m\right) u_{N}\right\rangle>0,
$$

in view of Lemma 2.1 under the assumption (1.10). We start with the following lemma.

Lemma 3.1. For $m>0$, assume that $K(x)$ satisfies (1.9), (1.10) and $K(x)=1-o(|x|) \geq 0$ as $|x| \rightarrow 0$. Define

$$
\varepsilon_{N}^{-1}:=\int_{\mathbb{R}^{2}}\left|(-\Delta)^{1 / 4} u_{N}\right|^{2} d x>0,
$$

where $u_{N}$ is a positive minimizer of (1.1) with $N<N^{*}$. Then we have

(i). $\varepsilon_{N}>0$ satisfies

$$
\varepsilon_{N} \rightarrow 0 \text { as } N \nearrow N^{*},
$$

and the Lagrange multiplier $\mu_{N}$ in (3.1) satisfies

$$
\mu_{N} \cdot \varepsilon_{N} \rightarrow \frac{1}{N^{*}} \text { as } N \nearrow N^{*} .
$$


(ii). Defining

$$
\bar{w}_{N}(x):=\varepsilon_{N}^{\frac{3}{2}} u_{N}\left(\varepsilon_{N} x\right),
$$

then

$$
\left\|(-\Delta)^{1 / 4} \bar{w}_{N}\right\|_{2}^{2}=1, \int_{\mathbb{R}^{3}}\left(\frac{K\left(\varepsilon_{N} x\right)}{|x|} *\left|\bar{w}_{N}\right|^{2}\right)(x)\left|\bar{w}_{N}(x)\right|^{2} d x \rightarrow 2 \text { as } N \nearrow N^{*},
$$

(iii). There exist a sequence $\left\{y_{N}\right\} \subset \mathbb{R}^{3}, R>0$ and $\eta>0$ such that

$$
\liminf _{N \nearrow N^{*}} \int_{B_{R}\left(y_{N}\right)}\left|\bar{w}_{N}\right|^{2} d x \geq \eta>0 .
$$

Proof. (i). On the contrary, if (3.4) is false, then $\left\{u_{N}(x)\right\}$ is bounded uniformly in $H^{\frac{1}{2}}\left(\mathbb{R}^{3}\right)$ as $N \nearrow N^{*}$. Setting

$$
\tilde{u}_{N}=\sqrt{\frac{N^{*}}{N}} u_{N}
$$

then $\left\{\tilde{u}_{N}\right\}$ is also bounded uniformly in $H^{\frac{1}{2}}\left(\mathbb{R}^{3}\right)$ and $\int_{\mathbb{R}^{3}}\left|\tilde{u}_{N}\right|^{2} d x=N^{*}$. Moreover, we deduce from Remark 2.1 that

$$
-m N^{*} \leq e\left(N^{*}\right) \leq \lim _{N \nearrow N^{*}} \mathcal{E}\left(\tilde{u}_{N}\right)=\lim _{N \nearrow N^{*}} \mathcal{E}\left(u_{N}\right)=\lim _{N \nearrow N^{*}} e(N)=-m N^{*} .
$$

This implies that $\left\{\tilde{u}_{N}\right\}$ is a minimizing sequence of $e\left(N^{*}\right)=-m N^{*}<0$. Similar to the proof of Theorem 1.1, by using (1.10) one can employ the concentration-compactness principle to deduce that there exist $u_{0} \in H^{\frac{1}{2}}\left(\mathbb{R}^{3}\right)$, a sequence $\left\{N_{k}\right\}$ with $N_{k} \nearrow N^{*}$ as $k \rightarrow \infty$ and $\left\{z_{k}\right\} \subset \mathbb{R}^{3}$ such that

$$
\tilde{u}_{N_{k}}\left(\cdot+z_{k}\right) \rightarrow u_{0}(\cdot) \text { strongly in } H^{\frac{1}{2}}\left(\mathbb{R}^{3}\right) \text { as } k \rightarrow \infty,
$$

and

$$
\lim _{k \rightarrow \infty} \int_{\mathbb{R}^{3}}\left(\frac{K(x)}{|x|} *\left|\tilde{u}_{N_{k}}\right|^{2}\right)(x)\left|\tilde{u}_{N_{k}}(x)\right|^{2} d x=\int_{\mathbb{R}^{3}}\left(\frac{K(x)}{|x|} *\left|u_{0}\right|^{2}\right)(x)\left|u_{0}(x)\right|^{2} d x .
$$

This indicates that $u_{0}$ is a minimizer of $e\left(N^{*}\right)$, which however contradicts Theorem 1.1 on the nonexistence of minimizers for $e\left(N^{*}\right)$ under the assumption (1.10). Therefore, (3.4) is proved.

We next prove (3.5) as follows. Since

$$
(-\Delta)^{\frac{1}{2}} \leq \sqrt{-\Delta+m^{2}} \leq(-\Delta)^{\frac{1}{2}}+m
$$

then

$$
\left\langle u_{N},(-\Delta)^{\frac{1}{2}} u_{N}\right\rangle \leq\left\langle u_{N}, \sqrt{-\Delta+m^{2}} u_{N}\right\rangle \leq\left\langle u_{N},(-\Delta)^{\frac{1}{2}} u_{N}\right\rangle+m N .
$$

We thus deduce from (3.3) and (3.4) that

$$
\lim _{N \nearrow N^{*}}\left\langle u_{N}, \sqrt{-\Delta+m^{2}} u_{N}\right\rangle \cdot \varepsilon_{N}=1 .
$$

Together with (3.2) and Remark 2.1, this yields that

$$
N \mu_{N} \cdot \varepsilon_{N}=-2 e(N) \cdot \varepsilon_{N}+\left\langle u_{N},\left(\sqrt{-\Delta+m^{2}}-m\right) u_{N}\right\rangle \cdot \varepsilon_{N} \rightarrow 1 \text { as } N \nearrow N^{*},
$$

which then gives (3.5).

(ii). By (3.3) and (3.6), we obtain that

$$
\left\|(-\Delta)^{1 / 4} \bar{w}_{N}\right\|_{2}^{2}=\varepsilon_{N} \cdot\left\|(-\Delta)^{1 / 4} u_{N}\right\|_{2}^{2}=1 .
$$


Moreover, since

$$
\frac{1}{2} \int_{\mathbb{R}^{3}}\left(\frac{K(x)}{|x|} *\left|u_{N}\right|^{2}\right)(x)\left|u_{N}\right|^{2} d x=\left\langle u_{N}, \sqrt{-\Delta+m^{2}} u_{N}\right\rangle-e(N)-m N,
$$

it follows from (3.4) and (3.10) that

$$
\frac{\varepsilon_{N}}{2} \int_{\mathbb{R}^{3}}\left(\frac{K(x)}{|x|} *\left|u_{N}\right|^{2}\right)(x)\left|u_{N}(x)\right|^{2} d x \rightarrow 1 \text { as } N \nearrow N^{*} .
$$

By (3.6), equivalently we have

$$
\frac{1}{2} \int_{\mathbb{R}^{3}}\left(\frac{K\left(\varepsilon_{N} x\right)}{|x|} *\left|\bar{w}_{N}\right|^{2}\right)(x)\left|\bar{w}_{N}(x)\right|^{2} d x \rightarrow 1 \text { as } N \nearrow N^{*},
$$

which then gives (3.7) by applying (3.11).

(iii). On the contrary, suppose that (3.8) is false. Then for any $R>0$, there exists a subsequence $\left\{\bar{w}_{N_{k}}\right\}$ with $N_{k} \nearrow N^{*}$ such that

$$
\lim _{k \rightarrow \infty} \sup _{y \in \mathbb{R}^{2}} \int_{B_{R}(y)}\left|\bar{w}_{N_{k}}\right|^{2} d x=0 .
$$

A proof similar to that of $[9$, Lemma A.2] thus yields that

$$
\int_{\mathbb{R}^{3}}\left(\frac{K\left(\varepsilon_{N_{k}} x\right)}{|x|} *\left|\bar{w}_{N_{k}}\right|^{2}\right)(x)\left|\bar{w}_{N_{k}}(x)\right|^{2} d x \rightarrow 0 \text { as } N_{k} \nearrow N^{*},
$$

which however contradicts (3.7). Therefore, (3.8) holds and the proof is complete.

Recall from Remark 2.1 that the proof of Theorem 1.1 gives the estimate $e(N) \sim-m N^{*}$ as $N \nearrow N^{*}$. In what follows, we give the refined upper bound of $e(N)$ as $N \nearrow N^{*}$ under the additional assumption (1.15).

Lemma 3.2. For $m>0$, suppose $K(x)$ satisfies (1.9), (1.10) and (1.15) for $1<p \leq \infty$. Define for any $Q \in \mathcal{G}$,

$$
\lambda:=\int_{\mathbb{R}^{3}} \frac{|\widehat{Q}(k)|^{2}}{|k|} d k \text { and } \gamma:=\int_{\mathbb{R}^{3}} \int_{\mathbb{R}^{3}}|y|^{p-1} Q^{2}(x-y) Q^{2}(x) d y d x \in(0, \infty] .
$$

Then,

$$
-m N \leq e(N) \leq-m N+\frac{\ell+1}{\ell} \ell^{\frac{1}{\ell+1}}\left(\frac{\kappa_{p}}{2}\right)^{\frac{1}{\ell+1}}(1+o(1))\left(N^{*}-N\right)^{\frac{\ell}{\ell+1}} \text { as } N \nearrow N^{*},
$$

where $\ell=\min \{2, p\}-1>0$, and $\kappa_{p}>0$ satisfies

$$
\kappa_{p}=\left\{\begin{array}{lr}
\beta \gamma, & 1<p<2 ; \\
\beta \gamma+m^{2} \lambda, & p=2 ; \\
m^{2} \lambda, & 2<p \leq \infty .
\end{array}\right.
$$

Proof. The lower bound of (3.13) follows directly from (2.4). To prove the upper bound of $e(N)$, similar to the proof of Theorem 1.1, we use a trial function $u_{\tau}$ of the form (2.5) so that $\int_{\mathbb{R}^{3}}\left|u_{\tau}\right|^{2} d x=N$. Similar to the argument of (2.6), we then have 


$$
\begin{aligned}
e(N) \leq \mathcal{E}\left(u_{\tau}\right)= & -m N+\left\langle u_{\tau},\left(\sqrt{-\Delta+m^{2}}-\sqrt{-\Delta}\right) u_{\tau}\right\rangle \\
& +\left[\left\|(-\Delta)^{1 / 4} u_{\tau}\right\|_{2}^{2}-\frac{1}{2} \int_{\mathbb{R}^{3}}\left(\frac{1}{|x|} *\left|u_{\tau}\right|^{2}\right)(x)\left|u_{\tau}\right|^{2} d x\right] \\
& +\frac{1}{2} \int_{\mathbb{R}^{3}}\left(\frac{1-K(x)}{|x|} *\left|u_{\tau}\right|^{2}\right)(x)\left|u_{\tau}\right|^{2} d x \\
= & -m N+\frac{N\left(N^{*}-N\right) \tau}{N^{*}}+\left\langle u_{\tau},\left(\sqrt{-\Delta+m^{2}}-\sqrt{-\Delta}\right) u_{\tau}\right\rangle \\
& +\frac{N^{2} \tau}{2\left(N^{*}\right)^{2}} \int_{\mathbb{R}^{3}}\left(\frac{1-K\left(\frac{x}{\tau}\right)}{|x|} * Q^{2}\right)(x) Q^{2}(x) d x \\
= & -m N+\frac{N\left(N^{*}-N\right) \tau}{N^{*}}+I+I I .
\end{aligned}
$$

As for the term $I$, it follows from Plancherel's theorem that

$$
\begin{aligned}
I & =\left\langle u_{\tau},\left(\sqrt{-\Delta+m^{2}}-\sqrt{-\Delta}\right) u_{\tau}\right\rangle \\
& =\frac{N}{N^{*}} \int_{\mathbb{R}^{3}}|\widehat{Q}(k)|^{2}\left(\sqrt{\tau^{2} k^{2}+m^{2}}-\tau|k|\right) d k \\
& =\frac{N m^{2}}{N^{*} \tau} \int_{\mathbb{R}^{3}}|\widehat{Q}(k)|^{2} \frac{1}{\sqrt{k^{2}+m^{2} / \tau^{2}}+|k|} d k \\
& =\frac{N m^{2}}{N^{*} \tau} \int_{\mathbb{R}^{3}} \frac{|\widehat{Q}(k)|^{2}}{2|k|} d k+o\left(\tau^{-1}\right)=\frac{N m^{2} \lambda}{2 N^{*} \tau}+o\left(\tau^{-1}\right) \text { as } \tau \rightarrow \infty,
\end{aligned}
$$

where (3.12) is used in the last equality.

We next estimate the term $I I$ as follows. Consider $\alpha \in(0,1)$, which will be determined later. Then,

$$
\begin{aligned}
& \int_{\mathbb{R}^{3}}\left(\frac{1-K\left(\frac{x}{\tau}\right)}{|x|} * Q^{2}\right)(x) Q^{2}(x) d x \\
&= \int_{|x| \geq \tau^{\alpha}}\left(\frac{1-K\left(\frac{x}{\tau}\right)}{|x|} * Q^{2}\right)(x) Q^{2}(x) d x \\
&+\left\{\int_{|x|<\tau^{\alpha}|y| \geq 2 \tau^{\alpha}}+\int_{|x|<\tau^{\alpha}} \int_{|y|<2 \tau^{\alpha}}\right\}\left(\frac{1-K\left(\frac{y}{\tau}\right)}{|y|} Q^{2}(x-y) Q^{2}(x)\right) d y d x \\
&=I I_{1}+I I_{2}+I I_{3} .
\end{aligned}
$$

From (1.7) and (1.8), we see that

$$
\begin{aligned}
I I_{1} & \leq \int_{|x| \geq \tau^{\alpha}}\left(\frac{1}{|x|} * Q^{2}\right)(x) Q^{2}(x) d x \\
& \leq C \int_{|x| \geq \tau^{\alpha}}(1+|x|)^{-9} d x \leq C \tau^{-6 \alpha} \text { as } \tau \rightarrow \infty .
\end{aligned}
$$

If $|x|<\tau^{\alpha}$ and $|y| \geq 2 \tau^{\alpha}$, then

$$
|x-y| \geq|y|-|x| \geq \frac{|y|}{2} \rightarrow \infty \text { as } \tau \rightarrow \infty .
$$

This estimate and (1.7) imply that 


$$
\int_{|y| \geq 2 \tau^{\alpha}} \frac{1-K\left(\frac{y}{\tau}\right)}{|y|} Q^{2}(x-y) d y \leq C \int_{|y| \geq 2 \tau^{\alpha}} \frac{1}{|y|^{9}} d y \leq C \tau^{-6 \alpha},
$$

and therefore,

$$
I I_{2} \leq C \tau^{-6 \alpha} \int_{|x|<\tau^{\alpha}} Q^{2}(x) d x \leq C \tau^{-6 \alpha} \text { as } \tau \rightarrow \infty .
$$

As for $I I_{3}$, since $K(x)$ satisfies (1.15) and $0<\alpha<1$, we have

$$
\begin{aligned}
& \int_{|y|<2 \tau^{\alpha}} \frac{1-K\left(\frac{y}{\tau}\right)}{|y|} Q^{2}(x-y) d y \\
& \quad \leq(\beta+o(1)) \tau^{-p} \int_{|y|<2 \tau^{\alpha}}|y|^{p-1} Q^{2}(x-y) d y \text { as } \tau \rightarrow \infty .
\end{aligned}
$$

Thus for $\tau \rightarrow \infty$,

$$
\begin{aligned}
\frac{\tau^{p}}{(\beta+o(1))} \cdot I I_{3} \leq & \int_{|x|<\tau^{\alpha}} \int_{|y|<2 \tau^{\alpha}}|y|^{p-1} Q^{2}(x-y) Q^{2}(x) d y d x \\
= & \iint_{|x|<\tau^{\alpha}}|z-x|<2 \tau^{\alpha} \\
\leq & C \int_{|x|<\tau^{\alpha}} \int_{|z|<3 \tau^{\alpha}}\left(|z|^{p-1}+|x|^{p-1}\right) Q^{2}(z) Q^{2}(x) d z d x \\
\leq & C\left[\int_{|x|<\tau^{\alpha}} Q^{2}(x) d x \int_{|z|<3 \tau^{\alpha}}|z|^{p-1} Q^{2}(z) d z\right. \\
& \left.+\int_{|z|<3 \tau^{\alpha}} Q^{2}(z) d z \quad \int_{|x|<\tau^{\alpha}}|x|^{p-1} Q^{2}(x) d x\right] \\
\leq & C+\left\{\begin{array}{l}
C \tau^{(p-6) \alpha}, \quad 1<p \neq 6 ; \\
\alpha C \ln \tau, \quad p=6 .
\end{array}\right.
\end{aligned}
$$

This indicates that if $1<p<6$, then

$$
\lim _{\tau \rightarrow \infty} \int_{|x|<\tau^{\alpha}} \int_{|y|<2 \tau^{\alpha}}|y|^{p-1} Q^{2}(x-y) Q^{2}(x) d y d x=\gamma \in(0, \infty),
$$

where $\gamma$ is defined by (3.12). Therefore, it follows from (3.20) and (3.21) that for $\tau \rightarrow \infty$,

$$
I I_{3} \leq\left\{\begin{array}{lr}
(\beta \gamma+o(1)) \tau^{-p}, & 1<p<6 \\
C \tau^{-p} \ln \tau, & p=6 \\
C \tau^{(\alpha-1) p-6 \alpha}, & p>6 .
\end{array}\right.
$$

This together with (3.17), (3.18) and (3.19) implies that for $\tau \rightarrow \infty$,

$$
I I \leq C \tau^{-6 \alpha+1}+\left\{\begin{array}{lr}
\frac{N^{2}(\beta \gamma+o(1))}{2\left(N^{*}\right)^{2}} \tau^{-p+1}, & 1<p<6 \\
C \tau^{-5} \ln \tau, & p=6 \\
C \tau^{(\alpha-1) p-6 \alpha+1}, & 6<p \leq \infty .
\end{array}\right.
$$


Choose $\alpha \in\left(\frac{1}{3}, 1\right)$ so that $\tau^{-6 \alpha+1}=o\left(\tau^{-1}\right)$ as $\tau \rightarrow \infty$. We then conclude from the above that

$$
I I \leq \begin{cases}\frac{N^{2}(\beta \gamma+o(1))}{2\left(N^{*}\right)^{2}} \tau^{-p+1}, & 1<p \leq 2 \\ o\left(\tau^{-1}\right), & 2<p \leq \infty\end{cases}
$$

We are now ready to derive the upper bound of (3.13) by discussing separately the following three cases:

Case 1: $2<p \leq \infty$. In this case, it follows from (3.22) that $I I=o\left(\tau^{-1}\right)$ as $\tau \rightarrow \infty$. We then deduce from (3.15) and (3.16) that

$$
e(N) \leq \mathcal{E}\left(u_{\tau}\right) \leq-m N+\frac{N\left(N^{*}-N\right) \tau}{N^{*}}+\frac{N m^{2} \lambda}{2 N^{*} \tau}+o\left(\tau^{-1}\right) \text { as } \tau \rightarrow \infty
$$

Taking

$$
\tau=\left[\frac{m^{2} \lambda}{2\left(N^{*}-N\right)}\right]^{\frac{1}{2}} \rightarrow \infty \text { as } N \nearrow N^{*},
$$

we thus obtain from above that

$$
e(N) \leq-m N+\sqrt{2} m \lambda^{\frac{1}{2}}(1+o(1))\left(N^{*}-N\right)^{\frac{1}{2}} \text { as } N \nearrow N^{*},
$$

which then gives the upper bound of (3.13) for Case 1.

Case 2: $p=2$. In this case, we deduce from (3.15), (3.16) and (3.22) that

$$
\begin{aligned}
e(N) \leq \mathcal{E}\left(u_{\tau}\right) \leq & -m N+\frac{N\left(N^{*}-N\right) \tau}{N^{*}} \\
& +\frac{N m^{2} \lambda}{2 N^{*} \tau}+\frac{N^{2}(\beta \gamma+o(1))}{2\left(N^{*}\right)^{2} \tau}+o\left(\tau^{-1}\right) \text { as } \tau \rightarrow \infty .
\end{aligned}
$$

By taking

$$
\tau=\left[\frac{m^{2} \lambda+\beta \gamma}{2\left(N^{*}-N\right)}\right]^{\frac{1}{2}},
$$

so that $\tau \rightarrow \infty$ as $N \nearrow N^{*}$, we then have

$$
e(N) \leq-m N+\sqrt{2}\left(m^{2} \lambda+\beta \gamma\right)^{\frac{1}{2}}(1+o(1))\left(N^{*}-N\right)^{\frac{1}{2}} \text { as } N \nearrow N^{*},
$$

which gives the upper bound of (3.13) for Case 2.

Case 3: $1<p<2$. In this case, one can derive from (3.15), (3.16) and (3.22) that

$$
e(N) \leq-m N+\frac{N\left(N^{*}-N\right) \tau}{N^{*}}+\frac{N^{2}(\beta \gamma+o(1))}{2\left(N^{*}\right)^{2} \tau^{p-1}}+o\left(\tau^{1-p}\right) \text { as } \tau \rightarrow \infty .
$$

Taking

$$
\tau=\left[\frac{(p-1) \beta \gamma}{2\left(N^{*}-N\right)}\right]^{\frac{1}{p}},
$$

so that $\tau \rightarrow \infty$ as $N \nearrow N^{*}$, it thus follows that

$$
e(N) \leq-m N+\frac{p}{p-1}(p-1)^{\frac{1}{p}}\left(\frac{\beta \gamma}{2}\right)^{\frac{1}{p}}(1+o(1))\left(N^{*}-N\right)^{\frac{p-1}{p}} \text { as } N \nearrow N^{*},
$$

and the upper bound of (3.13) is therefore established.

Proof of Theorem 1.3. (1). We first prove (1.13) under the assumptions (1.9), (1.10) and $K(x)=1-o(|x|) \geq 0$ as $|x| \rightarrow 0$. For the sequence $\left\{y_{N}\right\}$ obtained in (3.8), in this case we set

$$
w_{N}(x):=\bar{w}_{N}\left(x+y_{N}\right)=\varepsilon_{N}^{\frac{3}{2}} u_{N}\left(\varepsilon_{N} x+\varepsilon_{N} y_{N}\right) .
$$


It then follows from (3.8) that there exist $R>0$ and $\eta>0$ such that

$$
\liminf _{N \nearrow N^{*}} \int_{B_{R}(0)}\left|w_{N}\right|^{2} d x \geq \eta>0 .
$$

Moreover, we deduce from (3.1) and (3.26) that $w_{N}$ satisfies

$$
\left(\sqrt{-\Delta+\varepsilon_{N}^{2} m^{2}}-\varepsilon_{N} m\right) w_{N}-\left(\frac{K\left(\varepsilon_{N} x\right)}{|x|} *\left|w_{N}\right|^{2}\right) w_{N}=-\mu_{N} \varepsilon_{N} w_{N} \text { in } \mathbb{R}^{3} .
$$

Note from (3.7) that $\left\{w_{N}\right\}$ is bounded uniformly in $H^{\frac{1}{2}}\left(\mathbb{R}^{3}\right)$. Thus, under the assumption (1.10) there exists a subsequence of $\left\{w_{N}\right\}$, still denoted by $\left\{w_{N}\right\}$, such that $w_{N} \rightarrow w_{0} \geq 0$ in $H^{\frac{1}{2}}\left(\mathbb{R}^{3}\right)$. In addition, it follows from (1.9), (3.5) and (3.28) that $w_{0}$ satisfies

$$
\sqrt{-\Delta} w_{0}-\left(\frac{1}{|x|} *\left|w_{0}\right|^{2}\right) w_{0}=-\frac{1}{N^{*}} w_{0} \text { in } \mathbb{R}^{3} .
$$

By (3.27), we have $w_{0} \not \equiv 0$ in $\mathbb{R}^{3}$, and thus

$$
0<\int_{\mathbb{R}^{3}}\left|w_{0}\right|^{2} d x \leq \liminf _{N \nearrow N^{*}} \int_{\mathbb{R}^{3}}\left|w_{N}\right|^{2} d x=N^{*} .
$$

On the other hand, it follows from (3.29) and the standard Pohozaev identity that

$$
\frac{1}{N^{*}} \int_{\mathbb{R}^{3}}\left|w_{0}\right|^{2} d x=\int_{\mathbb{R}^{3}}|k|\left|\widehat{w}_{0}\right|^{2} d k=\frac{1}{2} \int_{\mathbb{R}^{3}}\left(\frac{1}{|x|} *\left|w_{0}\right|^{2}\right)\left|w_{0}\right|^{2} d x .
$$

We thus derive from (1.3) and (3.30) that

$$
\frac{N^{*}}{2} \leq \frac{\int_{\mathbb{R}^{3}}|k|\left|\widehat{w}_{0}\right|^{2} d k \cdot \int_{\mathbb{R}^{3}}\left|w_{0}\right|^{2} d x}{\int_{\mathbb{R}^{3}}\left(\frac{1}{|x|} *\left|w_{0}\right|^{2}\right)\left|w_{0}\right|^{2} d x}=\frac{\int_{\mathbb{R}^{3}}\left|w_{0}\right|^{2} d x}{2} \leq \frac{N^{*}}{2} .
$$

This indicates that $w_{0}$ optimizes the Gagliardo-Nirenberg type inequality (1.3). Moreover, $w_{N}$ converges to $w_{0}$ strongly in $L^{2}\left(\mathbb{R}^{3}\right)$, and in fact strongly in $L^{p}\left(\mathbb{R}^{3}\right)$ for all $p \in[2,3)$. Further, since $w_{N}$ and $w_{0}$ satisfy (3.28) and (3.29), respectively, standard elliptic regular theory gives that

$$
w_{N} \rightarrow w_{0} \geq 0 \text { strongly in } H^{\frac{1}{2}}\left(\mathbb{R}^{3}\right) .
$$

Since $w_{0} \geq 0(\not \equiv 0)$ satisfies (3.29), it then follows from [6, Theorem 1.1], [19, Theorem 11.8] that $w_{0}>0$ and $w_{0}$ is radially symmetric about some point $y_{1} \in \mathbb{R}^{3}$. Set

$$
Q_{0}(x)=\left(N^{*}\right)^{\frac{3}{2}} w_{0}\left(N^{*}\left|x-y_{1}\right|\right) .
$$

Then, $Q_{0}(x)=Q_{0}(|x|)>0$ optimizes the inequality (1.3) and it follows from (3.29) and (3.31) that $Q_{0}$ satisfies (1.4). Thus we have $Q_{0}(x) \in \mathcal{G}$. We therefore conclude (1.13) with $y_{0}=-N^{*} y_{1}$ by applying (3.26), (3.32) and (3.33).

(2). Based on the conclusions of the above (1), the limits (1.16) and (1.17) can be derived, if Lemma 3.3 below holds under the additional assumption (1.15). Indeed, by taking $Q=Q_{0}$ for Lemma 3.2, where $Q_{0}$ is as in (3.33), if Lemma 3.3 is true we then derive from (3.13) that (3.34) below is indeed an equality, and therefore (1.17) holds. Furthermore, (1.16) and (1.19) also follow immediately if Lemma 3.3 holds. This completes the proof of Theorem 1.3.

For simplicity, as in (1.18) we next denote

$$
\lambda_{0}:=\int_{\mathbb{R}^{3}} \frac{\left|\widehat{Q}_{0}(k)\right|^{2}}{|k|} d k \text { and } \gamma_{0}:=\int_{\mathbb{R}^{3}}\left(|x|^{p-1} *\left|Q_{0}\right|^{2}\right)\left|Q_{0}\right|^{2} d x,
$$

where $Q_{0}>0$ is as that of (3.33). Under the assumptions (1.9), (1.10) and (1.15) for $1<p \leq \infty$, the rest of this section is to establish the following lemma by following those obtained in the proof of Theorem 1.3. 
Lemma 3.3. For $m>0$, suppose $K(x)$ satisfies (1.9), (1.10) and (1.15) for $1<p \leq \infty$. Then the energy e $(N)$ satisfies

$$
e(N) \geq-m N+\frac{\ell+1}{\ell} \ell^{\frac{1}{\ell+1}}\left(\frac{\hat{\kappa}_{p}}{2}\right)^{\frac{1}{\ell+1}}(1+o(1))\left(N^{*}-N\right)^{\frac{\ell}{\ell+1}} \text { as } N \nearrow N^{*},
$$

where $\ell=\min \{2, p\}-1>0$, and $\hat{\kappa}_{p}>0$ satisfies

$$
\hat{\kappa}_{p}=\left\{\begin{array}{lr}
\beta \gamma_{0}, & 1<p<2 ; \\
\beta \gamma_{0}+m^{2} \lambda_{0}, & p=2 ; \\
m^{2} \lambda_{0}, & 2<p \leq \infty .
\end{array}\right.
$$

Especially, the identity of (3.34) holds if and only if

$$
\varepsilon_{N} \approx\left\{\begin{array}{lc}
\frac{1}{N^{*}}\left(\frac{2}{(p-1) \beta \gamma_{0}}\right)^{\frac{1}{p}}\left(N^{*}-N\right)^{\frac{1}{p}}, & 1<p<2 ; \\
\frac{1}{N^{*}}\left(\frac{2}{m^{2} \lambda_{0}+\beta \gamma_{0}}\right)^{\frac{1}{2}}\left(N^{*}-N\right)^{\frac{1}{2}}, & p=2 ; \\
\frac{1}{N^{*}}\left(\frac{2}{m^{2} \lambda_{0}}\right)^{\frac{1}{2}}\left(N^{*}-N\right)^{\frac{1}{2}}, & 2<p \leq \infty ;
\end{array}\right.
$$

where $f \approx g$ denotes $f / g \rightarrow 1$ as $N \nearrow N^{*}$.

Proof. Under the assumptions of Lemma 3.3, all conclusions of (1) in the above proof of Theorem 1.3 are true. Further, note from (1.3) and (3.3) that

$$
\begin{aligned}
e(N)=\mathcal{E}\left(u_{N}\right)= & -m N+\left\langle u_{N}, \sqrt{-\Delta} u_{N}\right\rangle-\frac{1}{2} \int_{\mathbb{R}^{3}}\left(\frac{1}{|x|} *\left|u_{N}\right|^{2}\right)(x)\left|u_{N}\right|^{2} d x \\
& +\left\langle u_{N},\left(\sqrt{-\Delta+m^{2}}-\sqrt{-\Delta}\right) u_{N}\right\rangle \\
& +\frac{1}{2} \int_{\mathbb{R}^{3}}\left(\frac{1-K(x)}{|x|} *\left|u_{N}\right|^{2}\right)\left|u_{N}\right|^{2} d x \\
\geq & -m N+\frac{N^{*}-N}{N^{*}} \frac{1}{\varepsilon_{N}}+\underbrace{\left\langle u_{N},\left(\sqrt{-\Delta+m^{2}}-\sqrt{-\Delta}\right) u_{N}\right\rangle}_{\widehat{I}} \\
& +\frac{1}{2} \underbrace{\left.\int \frac{1-K(x)}{|x|} *\left|u_{N}\right|^{2}\right)\left|u_{N}\right|^{2} d x .}_{\overparen{\mathbb{R}^{3}}}
\end{aligned}
$$

By Plancherel's theorem, we deduce from (3.26), (3.32) and (3.33) that

$$
\begin{aligned}
\widehat{I} & =\int_{\mathbb{R}^{3}}\left|\widehat{w}_{N}(k)\right|^{2}\left(\sqrt{k^{2} / \varepsilon_{N}^{2}+m^{2}}-\frac{|k|}{\varepsilon_{N}}\right) d k \\
& =m^{2} \varepsilon_{N} \int_{\mathbb{R}^{3}}\left|\widehat{w}_{N}(k)\right|^{2} \frac{1}{\sqrt{k^{2}+m^{2} \varepsilon_{N}^{2}}+|k|} d k \\
& =\frac{m^{2}+o(1)}{2} \varepsilon_{N} \int_{\mathbb{R}^{3}} \frac{\left|\widehat{w}_{0}(k)\right|^{2}}{|k|} d k \\
& =\frac{m^{2}+o(1)}{2} N^{*} \varepsilon_{N} \int_{\mathbb{R}^{3}} \frac{\left|\widehat{Q}_{0}(k)\right|^{2}}{|k|} d k \text { as } N \nearrow N^{*},
\end{aligned}
$$

which gives the estimate of $\widehat{I}$. 
We next estimate the term $\widehat{I I}$ as follows. Similar to the proof of [6, Lemma 2.2], one can derive from (3.28) and (3.31) that there exists $C>0$, independent of $N$, such that

$$
\left|w_{N}(x)\right| \leq C(1+|x|)^{-4} \text { and }\left(\frac{1}{|x|} *\left|w_{N}\right|^{2}\right)(x) \leq C(1+|x|)^{-1} \text { in } \mathbb{R}^{3} .
$$

For simplicity, we next set

$$
Q_{N}(x):=\left(N^{*}\right)^{\frac{3}{2}} w_{N}\left(N^{*} x\right)=\left(N^{*} \varepsilon_{N}\right)^{\frac{3}{2}} u_{N}\left(N^{*} \varepsilon_{N} x+N^{*} \varepsilon_{N} y_{N}\right),
$$

so that $Q_{N}$ satisfies (3.39) and

$$
Q_{N}(x) \rightarrow Q_{0}\left(\left|x-\frac{y_{0}}{N^{*}}\right|\right) \text { strongly in } H^{\frac{1}{2}}\left(\mathbb{R}^{3}\right) \text { as } N \nearrow N^{*},
$$

in view of (3.32) and (3.33). By (3.40) we have

$$
\begin{aligned}
\widehat{I I} & =\frac{1}{N^{*} \varepsilon_{N}} \int_{\mathbb{R}^{3}}\left(\frac{1-K\left(N^{*} \varepsilon_{N} x\right)}{|x|} *\left|Q_{N}\right|^{2}\right)\left|Q_{N}\right|^{2} d x \\
& \geq \int_{|x| \leq \varepsilon_{N}^{-1 / 2}} \int_{|y| \leq 2 \varepsilon_{N}^{-1 / 2}} \frac{1-K\left(N^{*} \varepsilon_{N} y\right)}{\left|N^{*} \varepsilon_{N} y\right|}\left|Q_{N}(x-y)\right|^{2}\left|Q_{N}(x)\right|^{2} d y d x \\
& \geq\left(N^{*} \varepsilon_{N}\right)^{p-1}(\beta+o(1)) \int_{|x| \leq \varepsilon_{N}^{-1 / 2}} \int_{|y| \leq 2 \varepsilon_{N}^{-1 / 2}}|y|^{p-1}\left|Q_{N}(x-y)\right|^{2}\left|Q_{N}(x)\right|^{2} d y d x,
\end{aligned}
$$

where $\beta>0$ is defined by (1.15). Moreover, similar to the calculations of (3.20) (where $\tau>0$ is replaced by $\varepsilon_{N}^{-1}$ ), we deduce from (3.39) and (3.41) that if $1<p<6$,

$$
\begin{aligned}
& \lim _{N \nearrow N^{*}} \int_{|x| \leq \varepsilon_{N}^{-1}} \int_{|y| \leq 2 \varepsilon_{N}^{-1}}|y|^{p-1}\left|Q_{N}(x-y)\right|^{2}\left|Q_{N}(x)\right|^{2} d y d x \\
& =\int_{\mathbb{R}^{3}}\left(|x|^{p-1} *\left|Q_{0}\right|^{2}\right)\left|Q_{0}\right|^{2} d x=\gamma_{0}<\infty .
\end{aligned}
$$

Together with (3.42), this implies that for $1<p<6$,

$$
\widehat{I I} \geq \gamma_{0}(\beta+o(1))\left(N^{*} \varepsilon_{N}\right)^{p-1} \text { as } N \nearrow N^{*} .
$$

In what follows, we shall complete the proof by considering separately the following three different cases:

Case 1: $2<p \leq \infty$. In this case, we deduce from (3.37) and (3.38) that

$$
\begin{aligned}
e(N)+m N & \geq \frac{N^{*}-N}{N^{*} \varepsilon_{N}}+\frac{m^{2}+o(1)}{2} \lambda_{0} N^{*} \varepsilon_{N} \\
& \geq \sqrt{2}\left[\left(m^{2}+o(1)\right) \lambda_{0}\right]^{\frac{1}{2}}\left(N^{*}-N\right)^{\frac{1}{2}} \text { as } N \nearrow N^{*},
\end{aligned}
$$

and moreover, a simple calculation shows that the second inequality of (3.44) is an identity if and only if

$$
\varepsilon_{N} \approx \frac{1}{N^{*}}\left(\frac{2}{m^{2} \lambda_{0}}\right)^{\frac{1}{2}}\left(N^{*}-N\right)^{\frac{1}{2}} \text { as } N \nearrow N^{*}
$$

Case 2: $p=2$. In this case, we deduce from (3.37), (3.38) and (3.43) that

$$
\begin{aligned}
e(N)+m N & \geq \frac{N^{*}-N}{N^{*} \varepsilon_{N}}+\frac{m^{2}+o(1)}{2} \lambda_{0} N^{*} \varepsilon_{N}+\frac{\gamma_{0}}{2}(\beta+o(1)) N^{*} \varepsilon_{N} \\
& \geq \sqrt{2}\left(\lambda_{0} m^{2}+\beta \gamma_{0}+o(1)\right)^{\frac{1}{2}}\left(N^{*}-N\right)^{\frac{1}{2}} \text { as } N \nearrow N^{*},
\end{aligned}
$$


and the second inequality of (3.46) is an identity if and only if

$$
\varepsilon_{N} \approx \frac{1}{N^{*}}\left(\frac{2}{m^{2} \lambda_{0}+\beta \gamma_{0}}\right)^{\frac{1}{2}}\left(N^{*}-N\right)^{\frac{1}{2}} \text { as } N \nearrow N^{*} .
$$

Case 3: $1<p<2$. In this case, we derive from (3.37), (3.38) and (3.43) that

$$
\begin{aligned}
e(N)+m N & \geq \frac{N^{*}-N}{N^{*} \varepsilon_{N}}+\frac{m^{2}+o(1)}{2} \lambda_{0} N^{*} \varepsilon_{N}+\gamma_{0}(\beta+o(1))\left(N^{*} \varepsilon_{N}\right)^{p-1} \\
& \geq \frac{p}{p-1}(p-1)^{\frac{1}{p}}\left(\frac{\beta \gamma_{0}}{2}\right)^{\frac{1}{p}}(1+o(1))\left(N^{*}-N\right)^{\frac{p-1}{p}} \text { as } N \nearrow N^{*},
\end{aligned}
$$

and the second inequality of (3.48) is an identity if and only if

$$
\varepsilon_{N} \approx \frac{1}{N^{*}}\left(\frac{2}{(p-1) \beta \gamma_{0}}\right)^{\frac{1}{p}}\left(N^{*}-N\right)^{\frac{1}{p}} \text { as } N \nearrow N^{*} .
$$

Therefore, (3.34) and (3.35) now follow from (3.44), (3.46) and (3.48). Moreover, (3.45), (3.47) and (3.49) also imply that (3.36) is true. This completes the proof of Lemma 3.3.

\section{Conflict of interest statement}

We declare that we have no conflict of interest.

\section{Acknowledgements}

The authors are very grateful to the referee for many useful suggestions which lead to some improvements of the present paper. The first author thanks Prof. Robert Seiringer for helpful discussions on the whole paper and constant encouragements over the past few years. The first author also thanks Prof. Rupert L. Frank for useful comments on the ground states of (1.4). Y. J. Guo is partially supported by National Natural Science Foundation of China (11322104 and 11671394), and X. Y. Zeng is partially supported by National Natural Science Foundation of China (11501555).

\section{References}

[1] X. Cabré, Y. Sire, Nonlinear equations for fractional Laplacians, I: regularity, maximum principles, and Hamiltonian estimates, Ann. Inst. Henri Poincaré, Anal. Non Linéaire 31 (2014) 23-53.

[2] L. Caffarellia, L. Silvestre, An extension problem related to the fractional Laplacian, Commun. Partial Differ. Equ. 32 (2007) $1245-1260$.

[3] D.M. Cao, Y.M. Su, Minimal blow-up solutions of mass-critical inhomogeneous Hartree equation, J. Math. Phys. 54 (2013) 121511, http://dx.doi.org/10.1063/1.4850879.

[4] Y.B. Deng, Y.J. Guo, L. Lu, On the collapse and concentration of Bose-Einstein condensates with inhomogeneous attractive interactions, Calc. Var. Partial Differ. Equ. 54 (1) (2015) 99-118.

[5] A. Elgart, B. Schlein, Mean field dynamics of boson stars, Commun. Pure Appl. Math. 60 (4) (2007) 500-545.

[6] R. Frank, E. Lenzmann, On ground states for the $L^{2}$-critical boson star equation, 15 pages, arXiv:0910.2721v2, 2010.

[7] R. Frank, E. Lenzmann, L. Silvestre, Uniqueness of radial solutions for the fractional Laplacian, Commun. Pure Appl. Math. 69 (9) (2016) 1671-1726.

[8] J. Fröhlich, B.L.G. Jonsson, E. Lenzmann, Effective dynamics for boson stars, Nonlinearity 20 (5) (2007) 1031-1075.

[9] J. Fröhlich, B.L.G. Jonsson, E. Lenzmann, Boson stars as solitary waves, Commun. Math. Phys. 274 (1) (2007) 1-30.

[10] J. Fröhlich, E. Lenzmann, Blowup for nonlinear wave equations describing boson stars, Commun. Pure Appl. Math. 60 (11) (2007) 1691-1705.

[11] J. Fröhlich, T.-P. Tsai, H.-T. Yau, On the point-particle (Newtonian) limit of the non-linear Hartree equation, Commun. Math. Phys. 225 (2) (2002) 223-274.

[12] Y.J. Guo, R. Seiringer, On the mass concentration for Bose-Einstein condensates with attractive interactions, Lett. Math. Phys. 104 (2014) $141-156$

[13] Y.J. Guo, Z.Q. Wang, X.Y. Zeng, H.S. Zhou, Properties for ground states of attractive Gross-Pitaevskii equations with multi-well potentials, 23 pages, arXiv:1502.01839, 2015.

[14] Y.J. Guo, X.Y. Zeng, H.S. Zhou, Energy estimates and symmetry breaking in attractive Bose-Einstein condensates with ring-shaped potentials, Ann. Inst. Henri Poincaré, Anal. Non Linéaire 33 (3) (2016) 809-828.

[15] Y.J. Guo, X.Y. Zeng, H.S. Zhou, Blow-up solutions for two coupled Gross-Pitaevskii equations with attractive interactions, Discrete Contin. Dyn. Syst., Ser. A 37 (7) (2017) 3749-3786. 
[16] E. Lenzmann, Well-posedness for semi-relativistic Hartree equations of critical type, Math. Phys. Anal. Geom. 10 (1) (2007) $43-64$.

[17] E. Lenzmann, Uniqueness of ground states for pseudorelativistic Hartree equations, Anal. PDE 2 (1) (2009) 1-27.

[18] E.H. Lieb, Existence and uniqueness of the minimizing solution of Choquard's nonlinear equation, Stud. Appl. Math. 57 (2) (1976/77) 93-105.

[19] E.H. Lieb, M. Loss, Analysis, Graduate Studies in Mathematics, vol. 14, American Mathematical Society, Providence, RI, 2001.

[20] E.H. Lieb, H.-T. Yau, The Chandrasekhar theory of stellar collapse as the limit of quantum mechanics, Commun. Math. Phys. 112 (1) (1987) $147-174$.

[21] P.L. Lions, The concentration-compactness principle in the calculus of variations. The locally compact case, Part I, Ann. Inst. Henri Poincaré, Anal. Non Linéaire 1 (1984) 109-145; Part II: Ann. Inst. Henri Poincaré, Anal. Non Linéaire 1 (1984) 223-283.

[22] M. Struwe, Variational Methods: Applications to Nonlinear Partial Differential Equations and Hamiltonian Systems, Ergebnisse Math., vol. 34, Springer, 2008.

[23] J.F. Yang, J.G. Yang, Existence and mass concentration of pseudo-relativistic Hartree equation, preprint, 2016, 34 pages. 